\title{
56. EJECTION OF BODIES FROM THE SOLAR SYSTEM IN THE COURSE OF THE ACCUMULATION OF THE GIANT PLANETS AND THE FORMATION OF THE COMETARY CLOUD
}

\author{
V. S. SAFRONOV \\ O. Schmidt Institute of Physics of the Earth, Moscow, U.S.S.R.
}

\begin{abstract}
The theory of planetary accumulation leads quite definitely to the conclusion that the formation of Oort's cometary cloud is the result of ejection of bodies to the outermost parts of the solar system due to encounters with the giant planets during their growth. Uranus and Neptune could have grown to their present dimensions only if the initial mass of solid material in their zones were substantially larger than that of these planets. The relative velocities of the bodies were increased through perturbations by the planetary embryos, and on reaching the escape velocity they would start to be ejected. Our concept of this process differs from that suggested by Öpik by the assumption that Jupiter and Saturn accreted hydrogen, not in solid but in gaseous state, and by the introduction of a more effective mechanism for the interaction with several embryos. In their final stages the embryos ejected amounts of mass an order of magnitude higher than the amounts accreted. Most of the mass was ejected into interstellar space by Jupiter, but the cometary cloud was created mainly by Neptune. The mass of the cloud is estimated to be about three times that of the Earth.
\end{abstract}

Study of the process of accumulation of the giant planets leads to the conclusion that in the final stage of their growth they should by their gravitational attractions eject a considerable number of solid bodies from the solar system. As the result of perturbations by the stars nearest to the Sun a small fraction of these bodies would remain at the periphery of the solar system, forming there the cometary cloud described in the classical work of Oort $(1950,1951)$.

For a correct evaluation of the amount of matter ejected it is necessary to discuss also other important factors that accompanied the process of accumulation of the planets: dissipation of gas from the solar system, and accretion of gas by Jupiter and Saturn. So far, there has been no single theory that takes account of all these factors simultaneously. It is possible, however, to divide the whole process into individual stages and to review at each of these only one or two of the most important factors. The results of such a review are briefly described below.

\section{The Initial Stage in the Accumulation of the Giant Planets}

Initially the planetary embryos grew on account of the solid bodies and particles that collided with them. The embryo masses were smaller than both $m_{a}$, at which value the accretion of gas begins, and $m_{e}$, when the ejection of bodies from the solar system begins. The process of embryo growth is quantitatively described by Safronov (1969). On the basis of this, the initial mass of the preplanetary cloud can be taken as equal to 0.05 to $0.06 M_{\odot}$. Assuming that in the region of the giant planets about $1.5 \%$ of the mass of the cloud has condensed into solid particles, we find that the initial quantity of solid matter there was some 300 to $350 M_{\oplus}$, i.e., about 6 or 7 times the quantity 
of solid matter in the giant planets. If there were less cloud material, Uranus and Neptune, which consist only of solid matter, could not have grown to their present size.

Gas took no part in the accumulation process but decreased somewhat the relative velocities of the small bodies and thus accelerated the growth of embryos. The effect was especially great when the bodies were small. At the same time gas was dissipated from the solar system. Apparently, the principal cause of the dissipation was a flow of fast particles emitted by the forming Sun during the time of its high electromagnetic activity (Schatzman, 1967). Towards the end of the initial stage the mass of gas in the zones of Jupiter and Saturn decreased by one order of magnitude. By this time the embryos reached mass $m_{a}$, at which point they began to absorb the remaining gas. The critical value $m_{a}$ is expected to be between the masses of Mars and the Earth. The embryo of Jupiter grew to mass $m_{a}$ in less than $10^{8} \mathrm{yr}$. The Uranus and Neptune embryos grew much more slowly, and when they reached the mass $m_{a}$ there was no gas left in their zones. These planets therefore had no gas accretion stage. Their initial stage terminated when their embryos grew to mass $m_{e}$, and they began to eject bodies from the solar system. For Neptune $m_{e} \approx 0.1 \mathrm{M}_{\oplus}$, and the time of growth in the first stage was over $10^{9} \mathrm{yr}$.

\section{Accretion of Gas by Jupiter and Saturn}

According to the theory of stationary accretion of gas by gravitating bodies (Bondi and Hoyle, 1944; Bondi, 1952), the rate of increase in the mass $m$ of a body as a consequence of absorption of gas that initially occupied an infinite space uniformly can be written

$$
\frac{\mathrm{d} m}{\mathrm{~d} t}=\frac{2 \pi \alpha G^{2} m^{2} \rho_{\infty}}{\left(v^{2}+c^{2}\right)^{3 / 2}}=A m^{2} \rho_{\infty}
$$

where $\rho_{\infty}$ is the density of the gas far enough from the body that it is not perturbed by its gravitational field, $v$ is the velocity of the gas with respect to the body, $c$ is the thermal velocity of the molecules, and $1<\alpha<2$. Attempts to explain the formation of comets in the solar system by this mechanism (capture of interstellar matter by the Sun's gravitational field) are known to have failed, as have similar attempts to explain the formation of hot giant stars from main sequence stars. There are no reasons, however, for refusing to apply the accretion mechanism to the massive embryos of the giant planets.

Let $m_{g}$ be the mass and $U$ the volume of unexhausted gas in the whole zone of a planet. If the average density of this volume is taken in Equation (1), i.e. $\rho_{\infty}=m_{g} / U$, it can easily be found that the Jupiter embryo, with an initial mass equal to $M_{\oplus}$, would exhaust all the gas in its zone in $10^{4} \mathrm{yr}$. In this case the characteristic accretion time for the remaining gas is about 4 orders less than the characteristic dissipation time.

However, the accretion of gas by the planetary embryos would be considerably hindered by the rotation of the preplanetary cloud. As an embryo exhausted the gas near its orbit the space would have been filled by gas coming from other heliocentric distances. Because of the low viscosity the radial displacement of the gas occurred with 
constant angular momentum (with respect to the Sun). This was accompanied by the development of an additional force $\delta f$ tending to prevent the displacement $\delta R$ in the heliocentric distance $R$, and (for unit gas mass) equal to

$$
\delta f=-\frac{G M_{\odot}}{R^{3}} \delta R \text {. }
$$

This force caused a considerable decrease in the density $\rho$ of the gas in the direction of the embryo:

$$
\frac{\mathrm{d} \ln \rho}{\mathrm{d} R}=-\frac{\mu}{R T} \delta f=-\frac{1}{h}
$$

and

$$
\frac{h}{R} \approx 10^{-3} \frac{R}{\delta R}
$$

Hence, the planet embryo could absorb gas only from a part of its zone. At the boundary the rates of accretion and dissipation are equal and

$$
\delta R \approx 0.15 R \approx 0.3 \Delta R,
$$

where $\Delta R$ is the half-width of the planet zone. Consequently, the planet embryo could absorb only about one-third of the gas remaining in the planet zone by the beginning of accretion.

The rate of gas dissipation can be found from the initial mass $m_{g 0}$ of gas in the planet zone. With $m_{g 0}$ equal to ten times the mass of Jupiter the dissipation time $\tau_{d}$ (i.e., the time corresponding to a decrease in density by a factor $e$ ) of gas in the Jupiter zone equals several tens of millions of years and turns out to be only a little less than the time of growth of the embryo to mass $m_{a}$.

\section{Ejection of Bodies from the Solar System}

The relative velocities of the bodies are determined by their gravitational perturbations during their close encounters, and they increased with the growth of the largest bodies. A comparison of the energy of random motions acquired in approaches and lost in collisions allows us to express (Safronov, 1969) the mean velocity $v$ of the bodies in terms of the Keplerian circular velocity depending on mass $m$ and radius $r$ of the planet embryo (the largest body in the zone), namely,

$$
v^{2}=G m / \theta r,
$$

where $\theta$ is a dimensionless parameter of the order of several units. At some embryo mass $m_{0}$ the greatest velocities (somewhat greater than $v$ ) have reached the value $v_{0}=0.414 v_{c}$, where $v_{c}=\sqrt{ }\left(G M_{\odot} / R\right)$ is the Keplerian circular velocity of the embryo, and bodies whose vector $\mathbf{v}_{0}$ is directed along $\mathbf{v}_{c}$ escape from the solar system. Actually, the mean velocity $v_{E}$ of ejected bodies is slightly greater than $v_{0}$, and the ejection occurs when $\mathbf{v}_{E}$ gets inside the cone of vertex angle $2 \varphi$ and axis coinciding with the direction of $\mathbf{v}_{c}$ about the Sun:

$$
v_{E}=v_{c}\left[\sqrt{ }\left(1+\cos ^{2} \varphi\right)-\cos \varphi\right]=v_{c} u(\varphi) .
$$


The more effective the mechanism for the increase of the energy of the random motions of the bodies, the larger $\varphi$ becomes and the greater the ratio $\lambda$ of the mass of bodies ejected by the embryo to the mass of bodies that have coalesced with it.

We have found that in a revolving system of gravitating bodies the energy $v^{2} / 2$ of the relative motion per unit mass increases as the result of encounters in unit time by the amount

$$
\xi_{1}=\beta^{\prime \prime} v^{2} / \tau_{g},
$$

where $\tau_{g}$ is the relaxation time during which the relative velocity vector $\mathbf{v}$ is deflected on the average by an angle $\pi / 2$; the coefficient $\beta^{\prime \prime}$ is about 0.13 . If the system includes a planet embryo, essentially more massive than other bodies and thus making the principal contribution to $\tau_{g}$, Equation (8) would contain, instead of $\tau_{g}$, the effective time of relaxation $\tau_{g e} \approx \tau_{g}^{\prime} / 2$, where $\tau_{g}^{\prime}$ is the relaxation time dependent on all bodies but the embryo.

Let us introduce the following symbols: $m$ for the mass of a planet embryo, $m_{b}$ the mass of all other bodies in the planet zone, $m_{E}$ the mass of bodies ejected from the zone beyond the boundaries of the solar system, $\mathcal{F}_{2}$ the energy of random motions per unit mass per second lost in collisions, $\tau_{s}^{*}$ the average lifetime of a body prior to its collision with the embryo, $\tau_{g}^{*}$ the time for the deflection of $\mathbf{v}$ by the angle $\pi / 2$ in encounters with the embryo only.

The equation for the conservation of energy of random motions of the bodies can be written

$$
m_{b} \mathrm{~d} v^{2}+\left(v_{E}^{2}-v^{2}\right) \mathrm{d} m_{E}+\left(v_{m}^{2}-v^{2}\right) \mathrm{d} m=2 m_{b}\left(\xi_{1}-\xi_{2}\right) \mathrm{d} t,
$$

where $v_{m}^{2}$ is the mean square velocity of bodies colliding with the embryo $m$. Substituting for $v$ and $\xi_{1}$ from Equations (6) and (8), then taking $\mathrm{d} m / \mathrm{d} t=m_{b} / \tau_{s}^{*}$ and retaining only the most important terms, we obtain

$$
\lambda=\frac{\mathrm{d} m_{E}}{\mathrm{~d} m} \approx \frac{2 \beta^{\prime \prime} v^{2}}{v_{E}^{2}-v^{2}} \frac{\tau_{s}^{*}}{\tau_{g e}} \approx \frac{4 \beta^{\prime \prime} f}{\chi} \frac{v_{e}^{2}}{v_{E}^{2}-v^{2}}
$$

where $v_{e}^{2}=2 G m / r=2 \theta v^{2}, \chi=\tau_{g}^{\prime} / \tau_{g}^{*}$, and

$$
f \approx 2 \ln \frac{D_{m} v^{2}}{G m}-1 \approx 4
$$

$D_{m}$, of the order of the radius of the sphere of action of the embryo, is the maximum approach distance that contributes to $\tau_{g}^{*}$. With $v^{2} \approx v_{E}^{2} / 3, v_{E} \approx v_{c} / 2, \beta^{\prime \prime} \approx 0.13$, we have

$$
\lambda \approx \frac{10}{\chi} \frac{v_{e}^{2}}{v_{c}^{2}}
$$

For Jupiter $\lambda \approx 200 / \chi$. Saturn was the second principal perturbing body for the majority of the bodies of the Jovian zone. Since $\tau_{g}^{*} \propto 1 / \mathrm{fm}^{2}$, and with $D_{m} \propto r, f \approx 2 \ln \mathrm{cm}^{-2 / 3}$,

$$
\chi=\frac{\tau_{g}^{\prime}}{\tau_{g}^{*}} \approx \frac{\left(m / m^{\prime}\right)^{2}}{1+(4 / 3 f) \ln \left(m / m^{\prime}\right)} \frac{R^{\prime 2}}{R^{2}} \approx 20
$$


where primed quantities refer to Saturn. Consequently, for Jupiter $\lambda \approx 10$. The mass ejected by Jupiter was about a quarter of its own mass. Bodies with smaller velocities did not escape into Saturn's zone and for them $\chi$ was greater. Later, bodies from the Uranus and Neptune zones escaped into Jupiter's zone, and Jupiter effectively ejected them out of the solar system.

The value of $\lambda=\lambda^{\prime}$ for Saturn for bodies moving rapidly through the zones of both Jupiter and Saturn can easily be found from Equation (10) on the assumption that $\tau_{g e}^{\prime}=\tau_{g e}$ and $v=$ const. Then $\lambda^{\prime} \approx 0.6 \lambda$. For slower bodies $\lambda^{\prime}$ is slightly less. In distinction to Jupiter and Saturn, which ejected bodies at practically their present mass, Uranus and Neptune ejected them during the whole course of their growth. Since $\lambda \propto v_{e}^{2} \propto \mathrm{m}^{2 / 3}$, the mean value of $\lambda$ during this whole time is $\bar{\lambda} \approx \frac{3}{5} \lambda$, where $\lambda$ refers to their present mass.

This evaluation agrees well with the above value for the whole mass of solid matter ejected from the preplanetary cloud, i.e., $\sim 300 M_{\oplus}$, giving $\lambda \approx 6$. In Table I (column 4) are given the approximate values of the masses ejected by the giant planets on the assumption that $\lambda \propto\left(v_{e} / v_{c}\right)^{2}$ and allowing for the additional ejection of bodies arising from the later forming, more distant planets.

TABLE I

Masses ejected by the giant planets

\begin{tabular}{llrrllll}
\hline Planet & $\left(v_{e} / v_{c}\right)^{2}$ & $m_{p}$ & $m_{E}$ & $10^{3}\left(\mathrm{~d} m_{c} / \mathrm{d} m_{E}\right)_{\min }$ & $\left(m_{c}\right)_{\min }$ & $10^{3}\left(\mathrm{~d} m_{c} / \mathrm{d} m_{E}\right)$ & $m_{c}$ \\
\hline Jupiter & 21.4 & 9 & 100 & 1.2 & 0.12 & 2.3 & 0.2 \\
Saturn & 14.1 & 12 & 80 & 2.1 & 0.18 & 5.4 & 0.4 \\
Uranus & 10.4 & 14.6 & 50 & 4.2 & 0.2 & 13 & 0.6 \\
Neptune & 18.8 & 17.2 & 60 & 6.6 & 0.4 & 21 & 1.3 \\
\hline
\end{tabular}

Total mass of the cometary cloud $0.9 M_{\oplus}<m<2.5 M_{\oplus}$.

The mass of the solid material ejected to the outskirts of the solar system can also be estimated. The semimajor axis $a$ of the orbit of a body moving with relative velocity $\mathbf{v}_{E}$ directed at angle $\varphi$ to the circular velocity $\mathbf{v}_{c}$ at heliocentric distance $R$ is determined by the expression

$$
\frac{R}{a}=1-\frac{v_{E}^{2}}{v_{c}^{2}}-2 \frac{v_{E}}{v_{c}} \cos \varphi
$$

Hence

$$
\frac{R}{a_{1}}-\frac{R}{a_{2}}=-2 \frac{v_{E}}{v_{c}}\left(\cos \varphi_{1}-\cos \varphi_{2}\right) \approx-2 \frac{v_{E}}{v_{c}} \sin \varphi \Delta \varphi .
$$

The lower limit for the mass of the cometary cloud can be found immediately by assuming the ratio of the mass $\mathrm{d} m_{c}$ ejected to the periphery of the solar system (in orbits with semimajor axes between $a_{1}$ and $a_{2}$ ) to the mass $\mathrm{d} m_{E}$ ejected into interstellar space to be equal to the ratio of corresponding solid angles between the cones $\varphi_{1}$ and $\varphi_{2}$ and inside the cone $\varphi$ :

$$
\left(\frac{\mathrm{d} m_{c}}{\mathrm{~d} m_{E}}\right)_{\min }=\frac{-\sin \varphi \Delta \varphi}{1-\cos \varphi}=\frac{R v_{c}}{2 v_{E}(1-\cos \varphi)}\left(\frac{1}{a_{1}}-\frac{1}{a_{2}}\right) .
$$


Such an assumption is valid for relatively close encounters when the vector $\mathbf{v}$ is deflected by more than $2 \varphi$. In this case $\mathbf{v}$ can appear at any point of the cone $\varphi$ with equal probability.

For very elongated elliptical orbits $a$ is about half the aphelion distance. Without committing much error it can be assumed that the bodies with orbital semimajor axes between $a_{1}=20000 \mathrm{AU}$ and $a_{2}=80000 \mathrm{AU}$ would undergo considerable stellar perturbations but still remain in circumsolar orbits. The values of $\left(\mathrm{d} m_{c} / \mathrm{d} m_{E}\right)_{\min }$ and of the mass $\left(m_{c}\right)_{\mathrm{min}}$ ejected to the outskirts of the solar system are calculated from Equation (15) for $\varphi=35^{\circ}$ and given in columns 5 and 6 of Table I. In this case the lower limit for the mass of the whole cometary cloud is found to be about one Earth mass; this is three times greater than the present value as calculated by Oort $(1950,1951)$ from the frequencies of the appearance of new comets.

It is known, however, that distant encounters are of great importance in the transport of energy. For small deflections of the vector $\mathbf{v}$ the ratio $\mathrm{d} m_{c} / \mathrm{d} m_{E}$ would be significantly greater than that given by Equation (15). We shall not reproduce here the rather cumbersome expressions that take into account the contribution of distant encounters. An approximate estimate leads to the simple relation:

$$
\frac{\mathrm{d} m_{c}}{\mathrm{~d} m_{E}} \sim \frac{\Delta \varphi}{\varphi} \ln \frac{2 \varphi}{\psi_{1}},
$$

where $\psi_{1} \approx 2 G m / D_{m} v_{E}^{2}$ is the smallest deflection angle of $\mathbf{v}$, corresponding to the largest value of $D_{m}$ considered. The values $\mathrm{d} m_{c} / \mathrm{d} m_{E}$ and $m_{c}$ found from Equation (16), and assuming $D_{m}$ equal to twice the radius of the sphere of action of each planet, are given in the last two columns of Table I. The result depends only slightly (logarithmically) on $D_{m}$. The mass of the cometary cloud is thus found to be about 3 Earth masses - in good agreement with the revised value of the present mass given by Öpik (1970). Jupiter has ejected into interstellar space more material than any other planet, although about half of the mass of the cometary cloud was supplied by Neptune. Because of the very slow growth of Neptune (and for that matter Uranus) most of the bodies ejected by it would by then have lost a significant portion of their volatiles.

\section{References}

Bondi, H. and Hoyle, F.: 1944, Monthly Notices Roy. Astron. Soc. 104, 273.

Bondi, H.: 1952, Monthly Notices Roy. Astron. Soc. 112, 195.

Oort, J. H.: 1950, Bull. Astron. Inst. Neth. 11, 91.

Oort, J. H.: 1951, Observatory 71, 129.

Öpik, E. J.: 1970, Moon 1, 487.

Safronov, V. S.: 1969, Ehvolyutsiya Doplanetnogo Oblaka i Obrazovanie Zemli $i$ Planet, Nauka, Moscow.

Schatzman, E.: 1967, Ann. Astrophys. 30, 963. 